\title{
¿Cómo cambian las ideas de los estudiantes de Máster de Profesorado sobre una actividad práctica de campo tras su vídeo-análisis y discusión en pequeños grupos?
}

\author{
María José Sáez Bondía \\ Departamento de Didáctica de las Ciencias Experimentales, Facultad de Ciencias Humanas y de la \\ Educación, Universidad de Zaragoza, Huesca, España.msaezbo@unizar.es \\ ORCID: https:/ / orcid.org/0000-0002-8733-1501 \\ Ángel Luis Cortés Gracia \\ Departamento de Didáctica de las Ciencias Experimentales, Facultad de Educación, Universidad de \\ Zaragoza,ZZaragoza,España.acortes@unizar.es \\ ORCID: bttps:/ / orcid.org/0000-0002-7075-9683
}

[Recibido: 30 Agosto 2018. Revisado: 18 Noviembre 2018. Aceptado: 30 Diciembre]

\begin{abstract}
Resumen: Los trabajos prácticos de campo en la formación inicial del profesorado de secundaria deberían incluir actividades que fuesen más allá de la propia realización de experiencias aplicables en un aula. El presente trabajo analiza las ideas declaradas por un grupo-clase de 16 estudiantes del Máster de Profesorado en diferentes momentos de un conjunto de actividades prácticas. En él, responden a cuestiones sobre la secuencia de actividades realizada y su posible transposición a un aula de secundaria, tanto de forma individual como en pequeños equipos, y analizan sus actuaciones durante la realización de una práctica de identificación de árboles y arbustos. El objetivo del trabajo es conocer si el vídeo-análisis de la actividad y su discusión posterior favorecen la evolución del Conocimiento Didáctico del Contenido sobre las actividades de campo. Los resultados muestran un cambio de las ideas de los estudiantes hacia modelos más acordes con lo que, desde la investigación en Didáctica de las Ciencias, se propone cuando se habla de los trabajos prácticos de campo.
\end{abstract}

Palabras clave: Trabajo práctico de campo, formación inicial del profesorado, Educación Secundaria, análisis de grabaciones de vídeo

How do change pre-service teachers' ideas about a fieldwork activity after its video-analysis and discussion in small groups?

\begin{abstract}
Fieldwork activities in pre-service training of secondary school should include activities that go beyond the implementation of experiences that could be carried out in a secondary school. The present work analyzes the ideas declared by a classroom group of 16 secondary pre-service teachers in different moments of a set of practical activities. In it, the students answer questions about the sequence of activities carried out and their possible transposition to a secondary classroom. These questions are answered individually and in small teams. They also analyze their actions during the completion of a fieldwork activity based on the identification of trees and bushes. The objective of this work is to know if the video-analysis of the activity and its subsequent discussion favor the evolution of the Pedagogical Content Knowledge on the field activities. The results show a change of ideas of secondary pre-service teachers that are more in agreement with the recommendations given by the investigation about fieldwork activities.

Keywords: Fieldwork, pre-service teacher training, Secondary Education, analysis of video recordings

Para citar este artículo: Sáez Bondía, M.J., Cortés Gracia, A.L. (2019) ¿Cómo cambian las ideas de los estudiantes de Máster de Profesorado sobre una actividad práctica de campo tras su vídeo-análisis y discusión en pequeños grupos? Revista Eureka sobre Enseñanza y Divulgación de las Ciencias 16(2), 2602. doi: 10.25267/Rev_Eureka_ensen_divulg_cienc.2019.v16.i2.2602
\end{abstract}




\section{Introducción}

La formación de los futuros profesores de ciencias se enmarca bajo una serie de saberes incluidos dentro del Conocimiento Didáctico del Contenido (CDC) (Shulman 1986; Acevedo 2009). No obstante, estos saberes no son elementos aislados, sino que incluyen una serie de conocimientos (sobre el contexto, los contenidos y su didáctica, según el modelo integrador de Gess-Newsome 1999) que se relacionan entre sí a través de un proceso reflexivo (Nilsson 2008; Park y Oliver 2008).

Autores como Bhattachryya, Volk y Lumpe (2009) especifican que llevar a cabo secuencias de indagación con profesores en formación inicial de un modo similar a como se realizarían en un aula incrementa la confianza de los estudiantes, que "aprenden a hacer lo que no saben haciéndolo" (Perrenoud 2004). Sin embargo, la realización exclusiva de experiencias trasladables al aula de secundaria en este contexto no es suficiente ya que, en muchos casos, estas experiencias sirven para que los estudiantes aprendan sobre la temática abordada (construcción del conocimiento científico), quedando en un segundo plano los aspectos didácticos relativos a la propia actividad que permitirían desarrollar el CDC (Sáez Bondía y Cortés Gracia 2014). Así, la creación de ambientes donde se discuta sobre las actividades realizadas en el aula o la realización de análisis de grabaciones de vídeo ofrece la oportunidad a los profesores en formación de visualizar, analizar y perfeccionar la práctica docente antes, durante y después de su trabajo en el aula (Rich y Hannafin 2009), fomentando de este modo el desarrollo de su CDC (Acevedo 2009).

El presente trabajo, enmarcado bajo un estudio de caso (Yin 2014), se contextualiza en una secuencia de tareas sobre identificación de árboles y arbustos a través de una actividad práctica de campo con estudiantes de Máster de Profesorado. Dicha secuencia incluye momentos en los que los estudiantes realizan análisis de sus actuaciones y de las de sus compañeros.

El objetivo de este estudio es conocer si el vídeo-análisis de la puesta en marcha de una actividad práctica y su posterior discusión en una puesta en común favorece un cambio en las ideas de los estudiantes de máster, desde una visión centrada en aspectos científicos (conceptos de botánica, identificación de ejemplares) hacia modelos más acordes a lo que desde la investigación educativa se recomienda sobre los trabajos prácticos de campo y sobre la percepción, como futuros docentes, de la propia actividad. Para ello se utilizan las respuestas dadas por los estudiantes en determinados momentos de la secuencias a través de unos cuestionarios diseñados a tal efecto. En concreto, se analizan los cambios en las ideas declaradas sobre cinco focos de interés: 1) los objetivos de la secuencia de actividades realizadas; 2) las dificultades que detectan durante el desarrollo de la misma; 3) las estrategias de resolución que emplean para resolverlas; 4) las dificultades que tendrían los estudiantes de secundaria al llevar a cabo la misma secuencia y 5) el tipo de propuestas de mejora que plantean para trasladar la secuencia realizada a un aula de secundaria.

\section{Trabajos prácticos de campo y su enfoque en la formación inicial del profesorado}

Existen numerosos trabajos sobre propuestas de salidas de campo, sobre el supuesto potencial didáctico de las mismas en la Enseñanza de las Ciencias (Rickinson et al. 2004; Braund y Reiss 2006; Dillon et al. 2006; Behrendt y Franklin 2014) e incluso sobre su presencia en las publicaciones de investigación educativa (Amórtegui et al. 2016; Aguilera 2018). Sin embargo, no son tan abundantes los trabajos sobre cómo se aprende en ese tipo de prácticas, cómo se deben o pueden introducir estas actividades en la formación del profesorado o cómo este tipo de prácticas contribuyen a la reflexión sobre aspectos didácticos y/o al desarrollo de 
competencias profesionales, salvo contadas excepciones (Dickerson, Dawkins y Annetta 2007; Allen 2011; Brusi et al. 2011, Morag y Tal 2017, entre otros).

Son muchos los factores que afectan a los trabajos prácticos de campo y que hay que tener en consideración cuando se diseñan y realizan actividades de este tipo (DeWitt y Storksdieck 2008). Tomando como referencia un trabajo clásico relativo al aprendizaje de los estudiantes durante una salida, Orion y Hofstein (1994) especificaban los siguientes elementos: 1) los relativos a la enseñanza, como el lugar donde se sale y su relación con el currículo, las estrategias didácticas a emplear, las orientaciones dadas por los profesores y su calidad; 2) los relativos a la propia salida, tales como las paradas a realizar, la duración y atractivo del trayecto y las condiciones ambientales durante la misma y 3) los relativos a los estudiantes, en los cuales concluyen como influyentes sus conocimientos sobre la materia a trabajar en la salida (aspectos cognitivos), la familiarización con el lugar donde se va a realizar la salida (aspectos geográficos) y el número de paradas o la duración de la salida (aspectos psicológicos).

En cuanto la planificación, diseño y aplicación de una salida, Pedrinaci (2012) aportaba una serie de orientaciones como:

1) No plantear demasiados objetivos, ya que el trabajo de campo demanda una recogida de datos, un análisis y reflexión que son contrarios a un gran número de objetivos.

2) Trabajar antes y después de la salida.

3) Dosificar la información para ayudar a los estudiantes a contextualizar los problemas y evitar dar información sobre cuestiones que no han sido planteadas por los alumnos y

4) Evitar que el estudiante piense que hay una única solución válida para el problema y que está muy clara, ya que en ese caso, puede perder la motivación.

Respecto a la relación del tipo de salida con los contenidos curriculares, en el presente trabajo seleccionamos como temática la identificación de ejemplares de plantas de un entorno próximo. En lo referente a ésta, Bebbington (2005) analizó la habilidad para identificar plantas de futuros maestros y profesores durante un curso de Biología, detectando una baja capacidad para ello. Este autor enfatizaba la importancia de trabajar estos aspectos tanto en educación primaria como en secundaria. Así, en los primeros niveles, se recomienda como objetivo conocer los principales organismos y algunas estrategias para identificarlos, mientras que en educación secundaria estas actividades se conciben como un medio para poder llevar a cabo pequeñas investigaciones, por ejemplo sobre ecología, señalando que la identificación no es un fin en sí misma.

Las dificultades con las que se pueden encontrar los estudiantes cuando se enfrentan a la identificación de ejemplares son muy variadas. Sáez Bondía y Cortés Gracia (2012) establecen como principales las relacionadas con los conceptos de botánica, con el uso de determinados procedimientos en el proceso de identificación, como la toma de datos o la utilización de guías, o las vinculadas a las actitudes hacia la propia actividad o al modo en el que abord an los problemas. Sobre este último aspecto, la aparición de debates entre estudiantes, la presencia del profesor durante su resolución, el uso de fuentes de información o de conocimientos previos o adquiridos previamente durante la misma salida, son modos en los que se enfrentan a las citadas dificultades.

Las consideraciones sobre el lugar donde realizar la salida, el tipo de itinerario a seguir o el número de paradas a realizar son aspectos que también hay que tener presentes cuando se realiza una salida al campo ya que, dependiendo de éstos, aparecen una serie de factores con los que hay que contar: el tiempo del que se dispone, el coste económico, la meteorología, el grado de conocimiento del lugar, la posibilidad de que el alumnado trabaje con autonomía 
(Del Carmen y Pedrinaci 1997). En el caso que exponemos seleccionamos como lugar los jardines del campus universitario, emplazamiento que permite realizar una salida sin costes, mientras se mantienen los beneficios propios de las mismas (Behrendt y Franklin 2014).

Si nos centramos en la formación del profesorado sobre las salidas de campo, los factores mencionados deberían tenerse en consideración no solo para su aplicación como si se tratase de estudiantes de secundaria o primaria, sino también centrando la atención en aquellos aspectos que permitan desarrollar su Conocimiento Didáctico del Contenido (Olson, CoxPetersen y McComas 2001; Dickerson et al. 2007) permitiéndoles, de este modo, reflexionar sobre las consecuencias del diseño de una determinada salida, las dificultades con las que se podría encontrar el alumnado y ellos mismos como futuros profesores durante su desarrollo o los posibles cambios del diseño de la propuesta con el objetivo de mejorarla. Es decir, permitir que este tipo de estudiantes comprendan la importancia del papel del docente antes, durante y después de la salida (Dori y Herscovitz 2005).

Así, para favorecer estos ambientes de reflexión, en las últimas décadas se han utilizado diversas estrategias, como la realización de actividades de instrucción directamente en el campo (Dickerson et al. 2007; Bhattachryya et al. 2009); el uso de entrevistas personales y grabaciones (Smith 1991); la elaboración de textos escritos en diferentes formatos (Hatton y Smith 1999; Mena, García Rodríguez y Tillema 2012); el vídeo-análisis de la actuación de los profesores en formación inicial en el aula (Simon y Campbell 2012; Blomberg et al. 2014) o las discusiones grupales sobre aspectos vinculados a la enseñanza (Yoon y Kim 2010). En este sentido, algunos estudios recientes muestran cómo la visión profesional de los profesores en formación necesita ser conceptualizada en términos de qué tipos de conocimientos están involucrados en el proceso de desarrollo de tal visión y de qué forma el vídeo les proporciona información valiosa sobre situaciones de aula que les ayuda a enfrentarse a los retos profesionales y a la toma de decisiones (Blomberg, Stürmer y Seidel 2011). Analizar esos vídeos y participar en grupos de discusión brinda a los profesores en formación la oportunidad de ver contenidos del curso en la práctica, pero también permite el desarrollo de diferentes perspectivas sin reforzar nociones preconcebidas que puedan impedir un diálogo significativo (Hatch et al. 2016). El uso de distintas estrategias (incluidas prácticas de enseñanza ambiciosas y conceptos erróneos de los estudiantes) pueden servir como herramientas para fomentar la atención del profesorado en formación así como para la discusión posterior a la puesta en práctica de actividades científicas que redundan en el desarrollo de una reflexión crítica sobre la Enseñanza de las Ciencias (Benedict-Chambers 2016). En este trabajo tratamos de combinar diversas estrategias (propuesta de actividad práctica, grabaciones en vídeo, cuestionarios y discusión en grupo) para fomentar la reflexión del profesorado en formación desde la perspectiva de la Didáctica de las Ciencias.

\section{Metodología}

\section{Contexto de la investigación}

La secuencia de actividades prácticas a partir de la cual se analizó el cambio de ideas fue realizada con un grupo-clase de 16 alumnos que cursaban la asignatura de "Diseño, organización y desarrollo de actividades para el aprendizaje de la Biología y Geología" del Máster de Profesorado en la Universidad de Zaragoza.

Para la realización de la misma (ver tabla 1), el grupo-clase fue dividido en 3 equipos de trabajo (A, B y C). Inicialmente, cada uno de los equipos realizaba una práctica de identificación de ejemplares leñosos dentro del campus universitario de un modo similar a como se podría realizar con estudiantes de secundaria, pero en la que los materiales aportados 
por el profesor a cada equipo se asignaban en órdenes temporales diferentes y poseían algún tipo de limitación. Por ejemplo, es muy difícil que todos los ejemplares presentes en un jardín urbano (campus universitario) aparezcan en una guía de identificación adaptada al nivel escolar (Carrasquer et al. 2001, véase Sáez Bondía y Cortés Gracia 2014). Todo el proceso de identificación fue grabado en vídeo con la correspondiente autorización de todos los participantes tanto para la grabación como para la distribución interna de los datos. En cada equipo, un estudiante "experto" en botánica grababa toda la actividad en vídeo y no intervenía en las tareas de toma de datos e identificación. Tras la realización de la práctica de identificación, los estudiantes cumplimentaban un cuestionario abierto (véase tabla 2) sobre aspectos relativos a la salida, inicialmente de forma individual y posteriormente de forma grupal. En este último caso, se demandaba acordar las respuestas que consideraban más oportunas a partir de las dadas de forma individual. A continuación, las grabaciones completas de la actividad (sin edición previa) eran entregadas a los equipos en dispositivos CD/DVD para su visualización en el aula o en sus domicilios. Así, los estudiantes visualizaban y analizaban las grabaciones de sus acciones y las de otro de los equipos durante la identificación de ejemplares y contestaban, de nuevo, primero individualmente y después en grupo, a preguntas relativas a la actividad realizada y a su posible aplicación en un aula de secundaria (véase tabla 2). Todos estos documentos, junto con el proceso de identificación de los ejemplares eran recogidos en un informe final. En la tabla 1 se muestra un resumen con la secuencia llevada a cabo y los materiales empleados.

Tabla 1. Resumen de la secuencia práctica analizada (adaptado de Sáez Bondía y Cortés Gracia 2014 y 2016).

\begin{tabular}{|c|c|c|c|c|}
\hline \multirow{2}{*}{$\begin{array}{c}\text { Sesiones/ } \\
\text { tiempo }\end{array}$} & \multirow{2}{*}{ ¿Qué se trabaja? } & \multicolumn{3}{|c|}{ Materiales por equipos } \\
\hline & & A & $\mathrm{B}$ & $\mathrm{C}$ \\
\hline $\begin{array}{l}\text { Sesión } 1 \\
\text { (2 horas) }\end{array}$ & $\begin{array}{l}\text {-Explicación previa de la actividad. } \\
\text {-Distribución de equipos de trabajo. }\end{array}$ & \multicolumn{3}{|c|}{$\begin{array}{l}\text { Consentimiento por parte del alumnado para la realización de la } \\
\text { actividad }\end{array}$} \\
\hline \multirow{4}{*}{$\begin{array}{l}\text { Sesión } 2 \\
\text { (3 horas) }\end{array}$} & \multirow{4}{*}{$\begin{array}{l}\text {-Salida al campus universitario. } \\
\text {-Recogida de datos. } \\
\text { Identificación de ejemplares. } \\
\text {-Filmación por parte de los "cámaras" } \\
\text { (estudiantes expertos en identificación } \\
\text { de plantas que se ofrecieron como } \\
\text { voluntarios en los equipos A y B y un } \\
\text { investigador en el equipo C). }\end{array}$} & \multicolumn{3}{|c|}{ Campus universitario (primera hora y media) } \\
\hline & & $\begin{array}{l}\text {-Dos libros de } \\
\text { identificación de } \\
\text { ejemplares a través } \\
\text { de claves } \\
\text { dicotómicas. } \\
\text {-Cámara de video y } \\
\text { persona encargada } \\
\text { de la grabación }\end{array}$ & $\begin{array}{l}\text {-Dos libros de } \\
\text { identificación de } \\
\text { ejemplares a través } \\
\text { de claves } \\
\text { dicotómicas. } \\
\text { - Ficha de } \\
\text { observación. } \\
\text {-Cámara de video y } \\
\text { persona encargada } \\
\text { de la grabación }\end{array}$ & $\begin{array}{l}\text {-Ficha de } \\
\text { observación } \\
\text {-Glosario con } \\
\text { terminología } \\
\text { botánica. } \\
\text {-Cámara de video } \\
\text { y persona } \\
\text { encargada de la } \\
\text { grabación }\end{array}$ \\
\hline & & \multicolumn{3}{|c|}{ Aula (Junto con los materiales que tenían en el campo) } \\
\hline & & $\begin{array}{l}\text {-Ficha de } \\
\text { observación } \\
\text {-Acceso a búsqueda } \\
\text { de información a } \\
\text { través de internet. }\end{array}$ & $\begin{array}{l}\text {-Acceso a búsqueda } \\
\text { de información a } \\
\text { través de internet. }\end{array}$ & $\begin{array}{l}\text {-Dos libros de } \\
\text { identificación de } \\
\text { ejemplares a través } \\
\text { de claves } \\
\text { dicotómicas. } \\
\text {-Acceso a } \\
\text { búsqueda de } \\
\text { información a } \\
\text { través de internet. }\end{array}$ \\
\hline $\begin{array}{l}\text { Sesión } 3 \\
\text { (3 horas) }\end{array}$ & $\begin{array}{l}\text {-Finalización de la identificación. } \\
\text {-Completar las cuestiones planteadas } \\
\text { para tras la identificación (cuestionario } 1 \text {, } \\
\text { véase tabla 2). } \\
\text {-Explicación de tareas no presenciales y } \\
\text { distribución de grabaciones }\end{array}$ & \multicolumn{3}{|c|}{$\begin{array}{l}\text {-Cuestiones que deben completar tras la finalización de la actividad } \\
\text { (tanto de forma individual como en común en el que expondrán } \\
\text { los aspectos más relevantes percibidos en su grupo). } \\
\text {-Entrega de cuestiones para el análisis de las grabaciones (tanto el } \\
\text { autoanálisis como el análisis cruzado de forma individual y puesta } \\
\text { en común, cuestionario 2, véase tabla } 2 \text { ) y CD con grabaciones de } \\
\text { su actuación y la de otro grupo }\end{array}$} \\
\hline $\begin{array}{l}\text { Sesión no } \\
\text { presencial } \\
\text { (fecha de } \\
\text { entrega } 20 \\
\text { días tras la } \\
\text { última } \\
\text { sesión } \\
\text { presencial) }\end{array}$ & $\begin{array}{l}\text {-Análisis de las grabaciones de video } \\
\text { propias. } \\
\text {-Contestar a las cuestiones planteadas } \\
\text { (cuestionario 2, véase tabla 2). } \\
\text {-Finalizar informe de identificación de } \\
\text { ejemplares. } \\
\text { Todos los documentos se recogen en un } \\
\text { informe final }\end{array}$ & \multicolumn{3}{|c|}{$\begin{array}{l}\text {-Cuestiones planteadas en cuestionario } 2 \text {. } \\
-C D \text { preparado con su actuación durante el desarrollo de la } \\
\text { actividad y la del grupo asignado para analizar. }\end{array}$} \\
\hline
\end{tabular}


Tabla 2. Cuestionarios empleados a lo largo de la secuencia, código asignado a cada cuestionario (C1I a C2CC), preguntas concretas planteadas a los estudiantes, orden cronológico de cumplimentación (números del 1 al 6 entre paréntesis) y código de colores para los focos de interés.

\begin{tabular}{|c|c|}
\hline \multicolumn{2}{|c|}{ Cuestionario 1: Sobre la actividad de identificación de árboles y arbustos realizada en el campus } \\
\hline Preguntas individuales (1)(C1I) & Preguntas en la puesta en comuin (2)(C1C) \\
\hline 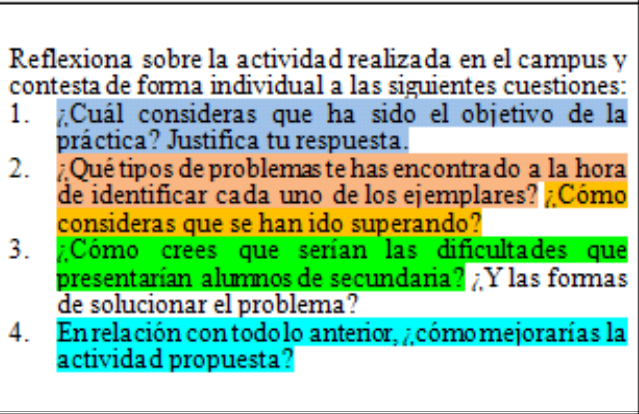 & $\begin{array}{l}\text { Una vez completados cada uno de los apartados, reúnete } \\
\text { con los miembros de tu grupopara hacer una puesta en } \\
\text { común. } \\
\text { 1. ¿Cuál es el principal objetivo de la práctica? } \\
\text { 2. Enumera los principales problemas que han surgido } \\
\text { durante la realización de la actividad. } \\
\text { 3. Enumera los principales modos de resolución de } \\
\text { problemas durante la realización de esta actividad. } \\
\text { 4. Enumera las principales dificultades previstas en los } \\
\text { 5. Indicad la principal propuesta de mejora para la } \\
\text { actividad y justificadlo }\end{array}$ \\
\hline \multicolumn{2}{|c|}{ Cuestionario 2: A partir del análisis de video de la actividad de identificación de árboles y arbustos } \\
\hline \multicolumn{2}{|c|}{ Parte 1: Autoanálisis } \\
\hline Preguntas individuales $(3)(C 2 A I)$ & Preguntas en la puesta en común $(5)(C 2 A C)$ \\
\hline $\begin{array}{l}\text { Tras haber visualizadolas grabaciones individualmente de } \\
\text { tu grupo, contesta a las siguientes cuestiones y justifica tu } \\
\text { respuestaindicando en qué momento del video se observa } \\
\text { (el minuto en el que aparece): } \\
\text { 1. ¿Qué tipo de problemas has observado al analizar la } \\
\text { actuación de tu grupo durante la práctica? } \\
\text { 2. ¿Cómo son las relaciones dentro del grupo? ¿Quétipo } \\
\text { de problemas aparecen durante la consecución de la } \\
\text { práctica al trabajar en grupo? }\end{array}$ & $\begin{array}{l}\text { Tras haber visualizado individualmente las grabaciones } \\
\text { tanto de tu grupo como de los compañeros del otro grupo } \\
\text { y una vez completados cada uno de los apartados de } \\
\text { formaindividual, reúnete con los miembros de tu grupo } \\
\text { para hacer una puesta en comín Contesta a las siguientes } \\
\text { cuestiones justificando tu respuesta e indicando en qué } \\
\text { momento del video se observa (el minuto en el que } \\
\text { aparece): } \\
\text { a)Relacionado contu grupo: }\end{array}$ \\
\hline $\begin{array}{l}\text { 3. ¿Cómo se solucionan los problemas que van } \\
\text { surgiendo? ¿Podrían haberse solucionado de otro } \\
\text { modo? }\end{array}$ & $\begin{array}{l}\text { 1. Indica y ejemplifica los dos problemas más } \\
\text { importantes quehan surgido durante la realización } \\
\text { de la actividad. }\end{array}$ \\
\hline $\begin{array}{l}\text { 4. ¿Cómo crees que hubiese sido la actuación de un } \\
\text { alumno de secundaria? } \\
\text { 5. Después de la discusiónen clase y de la visualización } \\
\text { del video, ¿ha cambiado tu opinión sobre el objetivo } \\
\text { de la práctica? }\end{array}$ & $\begin{array}{l}\text { 2. Enumeray ejemplificalos dos modos de resolución } \\
\text { de los problemas que surgen durante la realización } \\
\text { de esta actividad. } \\
\text { 3. Enumera los principales problemas previstos en los } \\
\text { alumnos/as de secundaria. }\end{array}$ \\
\hline \multicolumn{2}{|c|}{ Parte 2: Análisis de otro de los equipos } \\
\hline Preguntas individuales $(4)(\mathrm{C} 2 \mathrm{CI})$ & Preguntas en la puesta en común (6)(C2CC) \\
\hline $\begin{array}{l}\text { Tras visualizar las grabaciones de tus compañeros, } \\
\text { contesta a las siguientes cuestiones y justifica tu respuesta } \\
\text { indicando en qué momento del video se observa (el minuto } \\
\text { en el que aparece): }\end{array}$ & $\begin{array}{l}\text { b) Relaciona do con los compañeros del otro grupo: } \\
\text { 1. Indica y ejemplifica los dos problemas más } \\
\text { importantes quehan surgido durante la realización } \\
\text { de la actividad. }\end{array}$ \\
\hline $\begin{array}{l}\text { 1. ¿Qué tipo de problemas has observado al analizar la } \\
\text { actuación del grupo durante la práctica? } \\
\text { 2. ¿Cómo son las relaciones dentro del grupo? ¿Quétipo } \\
\text { de problemas aparecen durante la consecución de la } \\
\text { práctica al trabajar en grupo? }\end{array}$ & $\begin{array}{l}\text { 2. Enumeray ejemplifica los dos modos de resolución } \\
\text { de los problemas que surgen durante la realización } \\
\text { de esta actividad. } \\
\text { 3. Enumera los principales aspectos que cambiarias de } \\
\text { la actividad. }\end{array}$ \\
\hline $\begin{array}{l}\text { 3. ¿Cómo se solucionan los problemas que van } \\
\text { surgiendo? ¿Podrian haberse solucionado de otro } \\
\text { modo? }\end{array}$ & \\
\hline $\begin{array}{l}\text { 4. ¿Cuáles son los aspectos que más te llaman la } \\
\text { atención de la actuación del gnupo analizado? }\end{array}$ & \\
\hline $\begin{array}{l}\text { 5. Cómo serianlos problemas quetendrianlos alummos } \\
\text { de secundaria durante la realización de esta actividad }\end{array}$ & \\
\hline $\begin{array}{l}\text { a través de mo do en el que vuestros compañeros han } \\
\text { realiza do la actividad? }\end{array}$ & \\
\hline $\begin{array}{l}\text { 6. ¿Si tuvierais que llevar a cabo esta actividad en un } \\
\text { aula de secundaria, cómo lo haríais? }\end{array}$ & \\
\hline
\end{tabular}

Código de colores paralos focos de interés:

1. Objetivos de la actividad

2. Dificultades detectadas

3. Estrategias de resolución empleadas
4. Dificulta des previstas en los estudiantes de secundaria

5. Propuestas de mejora de la actividad 
Tanto el análisis de las grabaciones de sus actuaciones y las de sus compañeros como la puesta en común de las ideas expuestas individualmente, se incluyeron en la actividad con la intención de favorecer un cambio en las ideas de los estudiantes participantes. Según experiencias previas (Sáez Bondía y Cortés Gracia 2012), a través de la simple realización de la actividad práctica no pasaba de ser considerada como la mera reproducción y resolución formal a nivel científico de una actividad que se podría llevar a cabo en secundaria. Así se pretendía que pensasen en la actividad planteada como futuros profesores de secundaria considerando las recomendaciones expuestas en clase durante una sesión anterior. Del mismo modo, cambiar la secuencia temporal de uso de los recursos para cada uno de los equipos permitía visualizar la propuesta en su conjunto desde distintos enfoques.

\section{Instrumentos de registro de la información}

Las respuestas dadas en los cuestionarios recogidos en los informes finales fueron utilizadas para analizar la posible existencia de un cambio en las ideas de los estudiantes a lo largo de la secuencia (véase tabla 2). Hay que señalar que el tipo de cuestiones planteadas en cada momento se asociaban a los aspectos sobre los que se quería conocer el cambio de ideas, pero fueron formuladas con pequeñas diferencias para que los estudiantes no copiasen las respuestas dadas en los cuestionarios anteriores. Asimismo, se consideraron las grabaciones de vídeo y las anotaciones realizadas durante la identificación de los ejemplares. En la figura 1 se muestran los instrumentos de registro de la información utilizados y el momento de la actividad en el que fueron cumplimentados por los estudiantes.

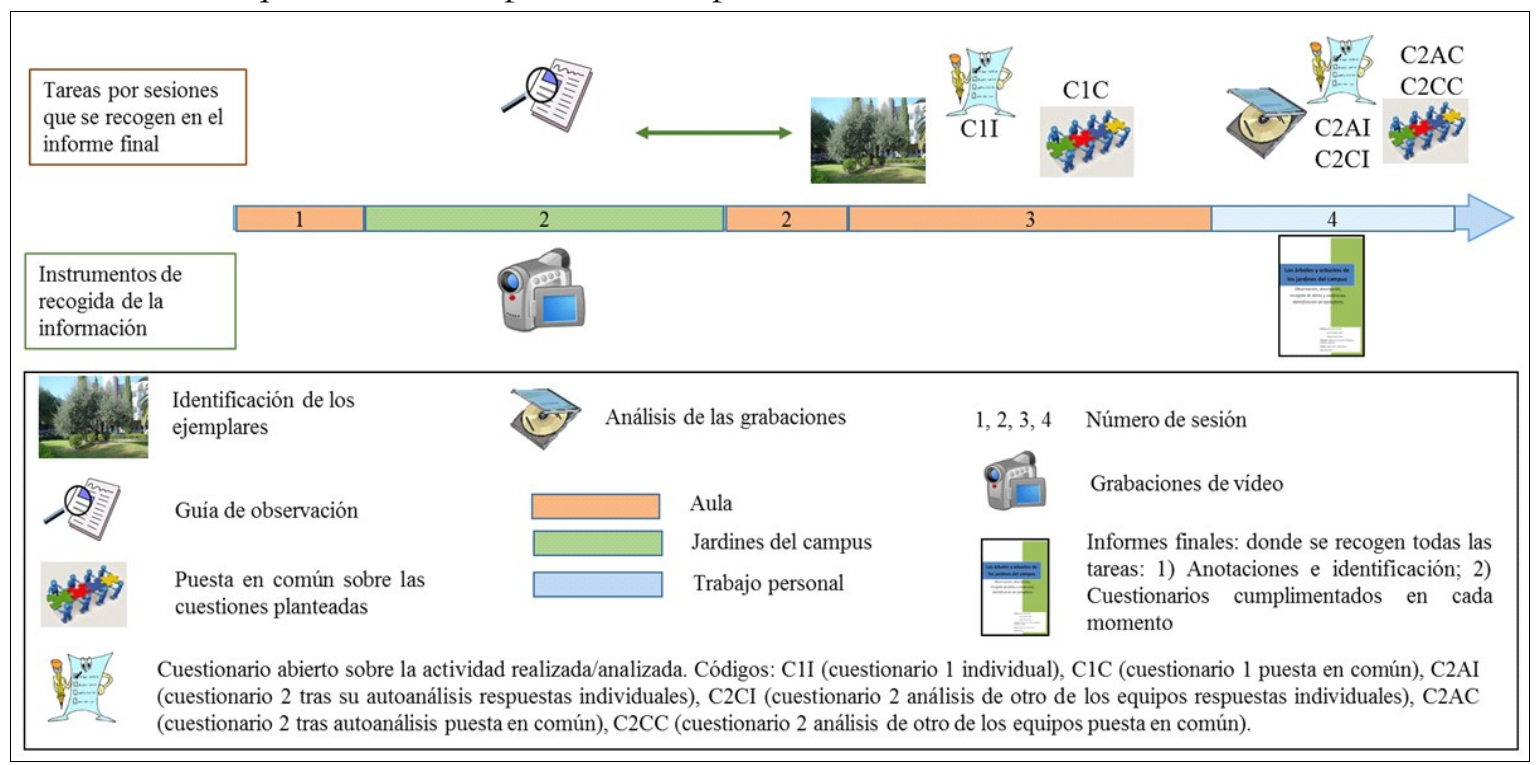

Figura 1. Instrumentos de recogida de la información en el caso.

\section{Análisis de los datos}

El análisis de los datos recogidos en este trabajo se plantea desde una perspectiva cualitativa. En el análisis de las respuestas dadas en los cuestionarios no se consideró la persona o equipo que respondía a la cuestión, sino el tipo de respuestas dadas en cada momento sobre cada uno de los cinco focos de interés del estudio: objetivos de la actividad, dificultades detectadas, estrategias de resolución, dificultades previstas en los estudiantes de secundaria y propuestas de mejora. De este modo, podíamos conocer cómo cambian las ideas cuando los estudiantes acordaban respuestas en equipo inicialmente (punto 1 de la figura 2), tras la visualización de las grabaciones (punto 2 de la figura 2) y cuando volvían a acordar respuestas dentro de cada equipo de trabajo (punto 3 de la figura 2). 
Sin embargo, no todas las respuestas relativas a los focos de interés fueron utilizadas para el análisis del caso. Las cuestiones realizadas tras la visualización de las grabaciones fueron agrupadas considerando tanto sus autoanálisis como los análisis cruzados (enmarcados en color verde en la figura 2). Así, de los cuestionarios C2CI y C2CC, solo fueron consideradas las respuestas relativas a las propuestas de mejora, ya que fueron planteadas únicamente tras la visualización de las actuaciones de sus compañeros. Sin embargo, las respuestas relativas a las dificultades y estrategias de resolución observadas en dichos cuestionarios fueron consideradas como una herramienta de validación (triangulación de datos, Simons 2011).

Asimismo, las cuestiones relativas a los objetivos percibidos a lo largo de la actividad no fueron incluidas en los cuestionarios C2AC y C2CC, por lo que no se estudió el cambio de ideas sobre ese foco de interés en ese momento.

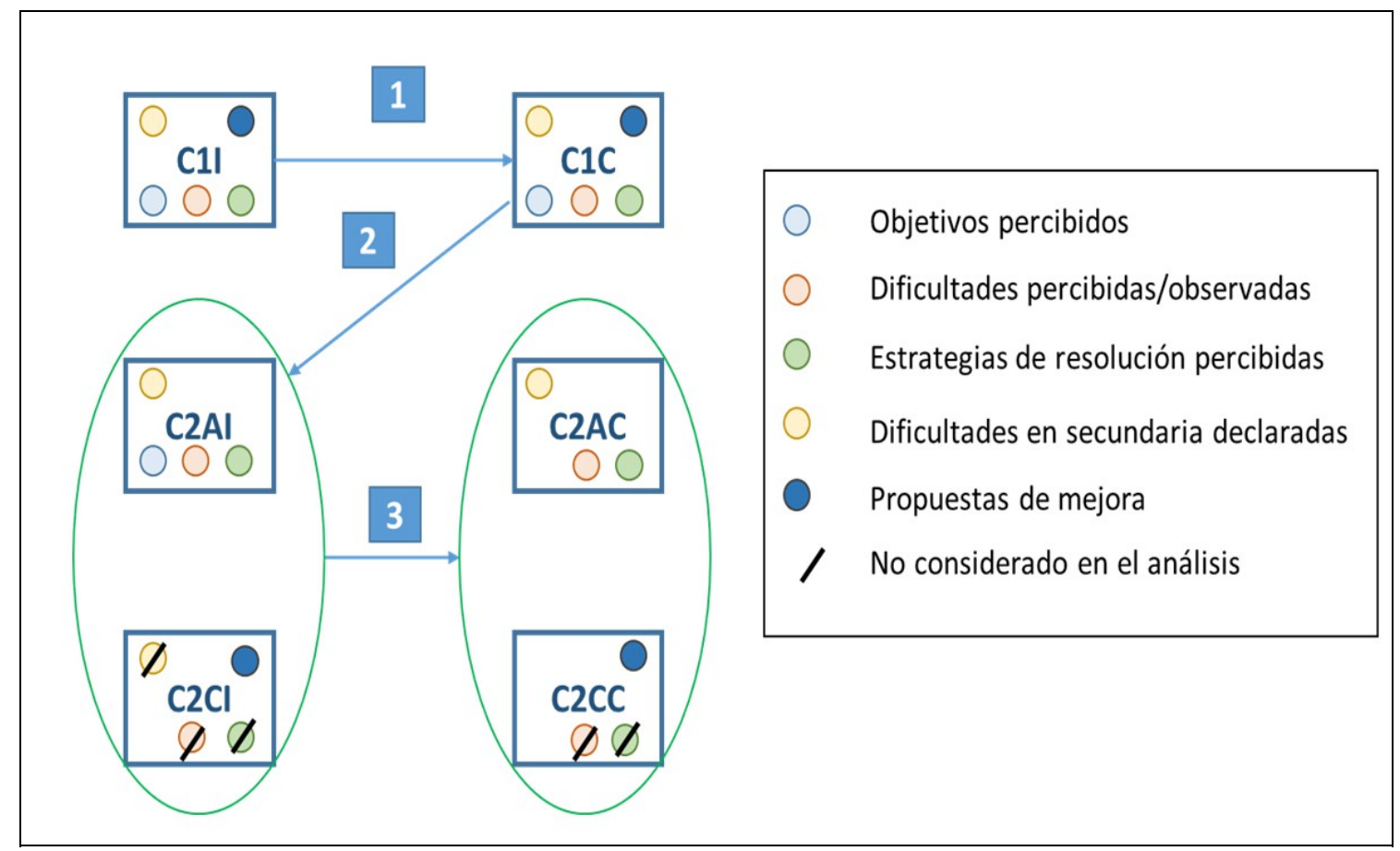

Figura 2. Comparaciones realizadas para estudiar los cambios en las ideas de los estudiantes a partir de los cuestionarios elaborados y los focos de interés (círculos coloreados). Véase codificación en figura 1.

A partir de las respuestas de los estudiantes se fue elaborando un sistema de categorías tal y como se muestra en la tabla 3 , tomando como referencia: 1) las dificultades con las que se encuentran estos estudiantes cuando realizan la actividad y las estrategias de resolución que emplean (Sáez Bondía y Cortés Gracia 2012), 2) los elementos que son necesarios para la realización de una salida (Orion y Hofstein 1994) y 3) los niveles de reflexión escrita para ver el modo en el que planteaban las propuestas de mejora (Hatton y Smith 1992). 
Tabla 3. Sistemas de categorías empleado para conocer las ideas de los estudiantes sobre la actividad planteada (adaptada de Sáez Bondía y Cortés Gracia 2016)

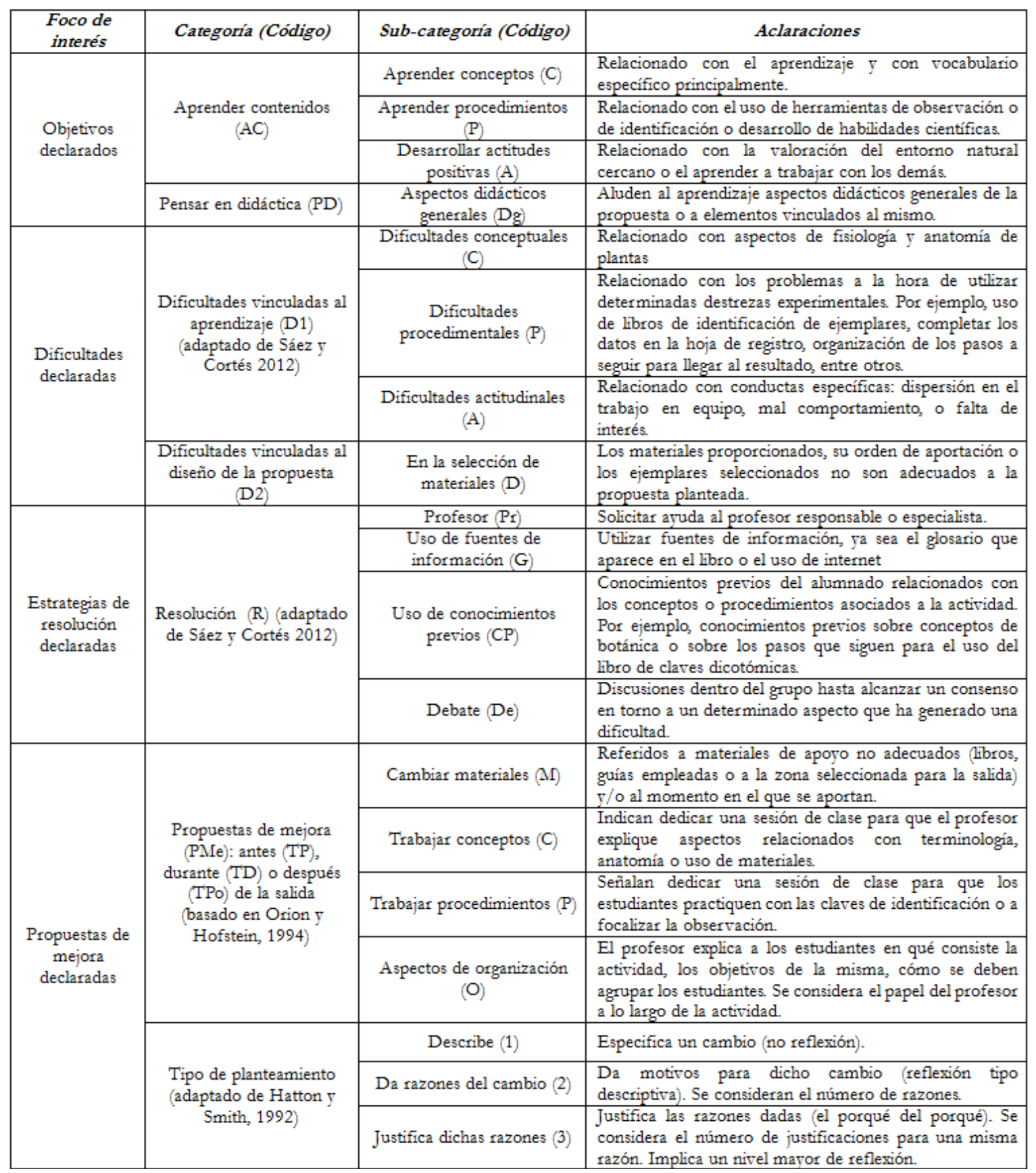

\section{Resultados}

En este apartado mostramos las declaraciones de los estudiantes en los cuatro momentos en los que cumplimentaron los cuestionarios. Hay que señalar que los estudiantes del equipo B entregaron directamente sus respuestas grupales tras la visualización de las grabaciones. No obstante, este hecho no altera los resultados generales, ya que el objetivo del caso es conocer los cambios de las ideas del grupo-clase estudiado en su conjunto. 
Declaraciones sobre los objetivos de la propuesta, las dificultades vividas, las estrategias de resolución y las posibles dificultades en alumnos de secundaria

La figura 3 muestra, para cada uno de los focos de interés, las sub-categorías que aparecen y su proporción, atendiendo al diámetro de cada uno de los círculos representados para los cuatro momentos de la secuencia de actividades, en los que se cumplimentaron los cuestionarios referidos a cada foco de interés. Sobre cada círculo se muestra el porcentaje de aparición respecto al total de respuestas de cada apartado.

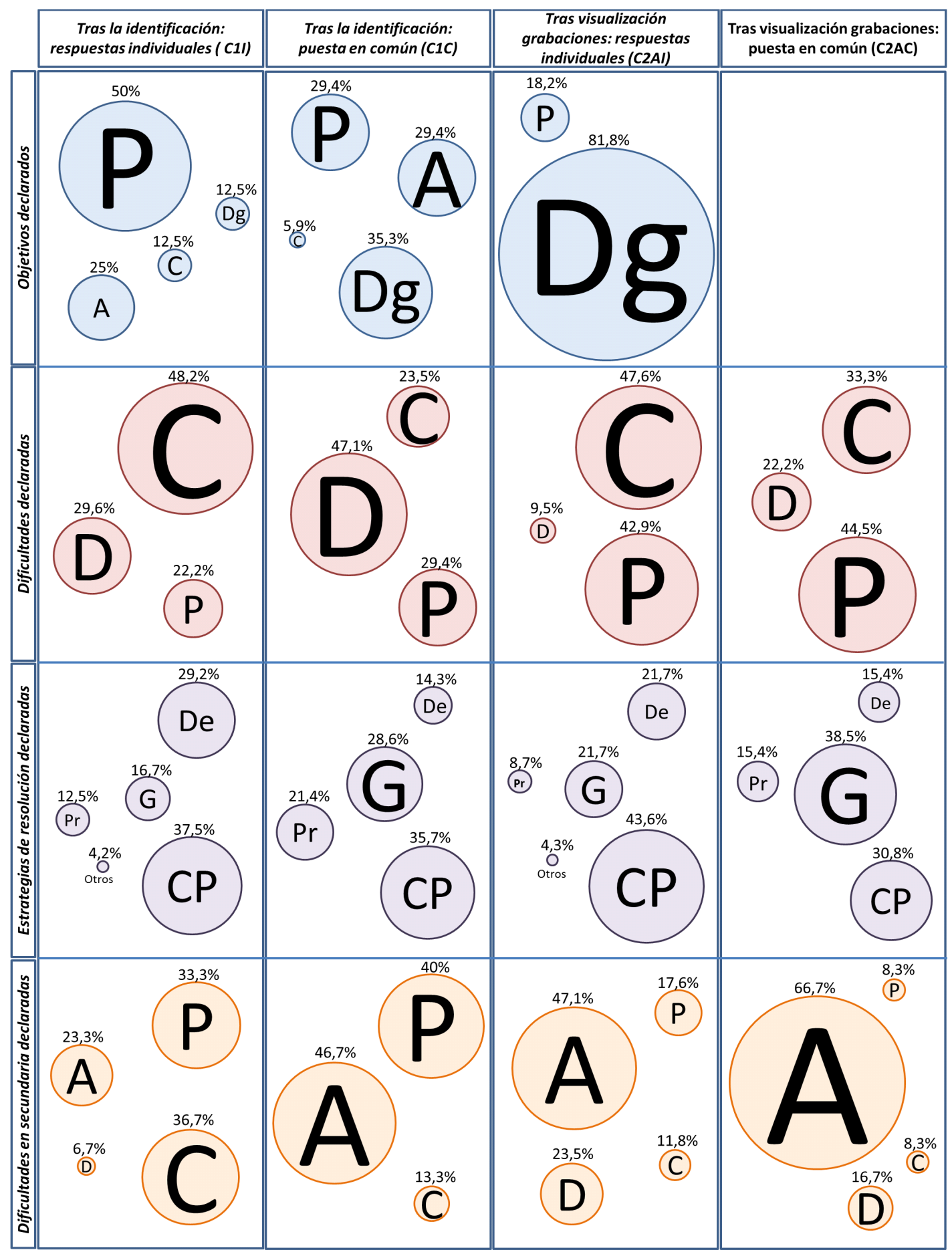

Figura 3. Ideas declaradas por los estudiantes en los cuatro momentos de la actividad. Códigos, véase sistema de categorías en la tabla 3. 
A continuación se describen los principales resultados para cada foco de interés analizado.

\section{Los objetivos declarados}

La percepción de los objetivos de la actividad por parte de los estudiantes cambia notablemente. Mientras que nada más finalizar la identificación de ejemplares declaran que los objetivos de la misma son, principalmente, aprender a trabajar en equipo (A) y a usar las claves dicotómicas o adquirir destrezas de observación (P), tras la puesta en común se incrementa el número de declaraciones relacionadas con aspectos didácticos de la propuesta (Dg). Dichos comentarios aluden, principalmente, a situarse en el papel de un estudiante de secundaria. Asimismo, la idea de que el objetivo de la actividad es el desarrollo de actitudes (A) también se incrementa, pero, en este caso, hacen referencia al desarrollo de autonomía en el trabajo.

Tras la visualización de las grabaciones, la percepción del objetivo se centra principalmente en ponerse en el papel de un estudiante de secundaria o pensar en los materiales a emplear cuando se hace la actividad (Dg), desapareciendo la percepción de objetivos relacionados con la adquisición de conceptos sobre botánica (C) o el desarrollo de actitudes (A).

En la tabla 4 mostramos algunos ejemplos de las declaraciones relativas a la categoría "Pensar en didáctica" (Dg) tras la visualización de las grabaciones (C2AI). Dentro de esta categoría nos encontramos con estudiantes que buscan aparentemente "recetas de cocina" y otros que visibilizan la propuesta desde una perspectiva de "múltiples posibilidades", en las que, dependiendo del contexto, todas pueden ser válidas. Asimismo, otros estudiantes relacionan el objetivo con las dificultades vividas.

Tabla 4. Ejemplos de objetivos declarados en C2AI relativos a la categoría Dg.

\begin{tabular}{|c|}
\hline Ejemplos de estudiantes que buscan "recetas de cocina" \\
\hline $\begin{array}{c}\text { Ejemplos de estudiantes que ven "múltiples posibilidades" } \\
\text { futuros alumnos" }\end{array}$ \\
\hline $\begin{array}{c}\text { "Creo que el objetivo de esta actividad es que seamos conscientes de que una misma actividad se puede } \\
\text { enfocar de maneras muy diferentes y, como docentes, tenemos que decidir que opción es mejor para nuestros } \\
\text { alumnos" }\end{array}$ \\
\hline Ejemplos de estudiantes que enfocan el objetivo desde las dificultades encontradas \\
\hline "Ponernos en la situación de los alumnos de secundaria al realizar esta actividad. Si nosotros tenemos \\
dificultades o no sabemos llegar a algún punto, ellos tampoco" \\
"Identificar los problemas que pueden surgir y anticiparnos en la medida de lo posible"
\end{tabular}

\section{El tipo de dificultades vividas y las estrategias de resolución empleadas}

Respecto al tipo de dificultades con las que declaran encontrarse en los cuatro momentos, se observan pequeñas variaciones. En las respuestas dadas por los equipos se incrementan las dificultades relacionadas con una "mala selección de materiales" (D: "u so de guías de campo específicas para un terreno concreto que no es el de la práctica realizada", ya que la guía presenta ejemplos de la ciudad de Teruel) o relacionadas con aspectos procedimentales (P: “... no se fijaban en las características que usa la guía para diferenciar las distintas especies, por ejemplo la longitud de la hoja, hojas en un mismo o en distinto plano, etc.") y se reducen las dificultades de tipo conceptual (C: "dificultad para comprender algunos conceptos que se usaban en la guía de identificación”).

Las declaraciones sobre las estrategias de resolución empleadas no varían prácticamente nada en los cuatro momentos. Se consideran como estrategias más relevantes el uso de guías (G: "comparando los dibujos con la realidad", “... esquemas y dibujos que ofrecía la guía", "utilización de las guías, glosario y dibujos"), ya sean las empleadas en la propia actividad u otras, y los conocimientos previos propios y/o ajenos (CP: "por algunos conocimientos 
previos que poseían personas del grupo", "recurrir a miembros del grupo con conocimientos de botánica superiores al resto"). Al igual que sucede con las dificultades, en las respuestas dadas en grupo se incrementan las categorías que individualmente aparecían en menos proporción y se reducen ligeramente aquellas que aparecían en mayor proporción.

\section{Las posibles dificultades en secundaria}

Las respuestas acerca de las dificultades que podrían tener los estudiantes de secundaria cambian considerablemente. Inicialmente, la ausencia de determinados ejemplares en el libro de identificación (D) no supondría apenas problemas para un alumno de secundaria. Al contrario ocurriría con aquellos aspectos relacionados con la pérdida de interés o las dificultades a la hora de trabajar en equipo (A). En la puesta en común se incrementan las referencias a dificultades de tipo actitudinal (A) y procedimental (P).

Tras la visualización de las grabaciones, el número de ocasiones en las que los estudiantes aluden a problemas relacionados con los materiales (D) se incrementa en general, mientras se reducen los asociados a conceptos $(\mathrm{C})$ o procedimientos $(\mathrm{P})$. Dicho cambio se mantiene tras la puesta en común, incrementando las declaraciones relativas a las dificultades actitudinales (A), aunque éstas lo hacen en un sentido diferente al de los momentos iniciales. En general, las respuestas antes de la visualización de las grabaciones son más generales (y tópicas), mientras que tras la visualización de las grabaciones se hacen más concretas tal y como se muestra en el ejemplo de la tabla 5 .

Así, las dificultades actitudinales declaradas pasan de aspectos que culpabilizan, en cierta medida, a la edad de los estudiantes, a aquellos que se relacionan con el diseño de la propia actividad (desinterés por frustración ante la posibilidad de no llegar a identificar el ejemplar con los materiales aportados). Estas declaraciones son coherentes con el incremento en las respuestas dadas sobre las dificultades vinculadas a un mal diseño de la actividad (D), que apenas son declaradas en los momentos iniciales.

Tabla 5. Ejemplo de respuestas sobre dificultades actitudinales de alumnos de secundaria por momentos.

\begin{tabular}{|c|c|c|c|}
\hline $\begin{array}{l}\text { Respuesta inicial } \\
\text { (cuestionario C1I) }\end{array}$ & $\begin{array}{l}\text { Puesta en común inicial } \\
\text { (C1C) }\end{array}$ & $\begin{array}{l}\text { Tras la visualización de } \\
\text { las grabaciones (C2AI) }\end{array}$ & $\begin{array}{l}\text { Puesta en común final } \\
(C 2 A C)\end{array}$ \\
\hline $\begin{array}{l}\text { "Pueden distraerse de la } \\
\text { actividad al estar } \\
\text { trabajando en equipo, irse } \\
\text { a hablar de otros temas, } \\
\text { relacionados o no con la } \\
\text { actividad" }\end{array}$ & $\begin{array}{l}\text { "Debido a su edad, } \\
\text { creemos que los grupos } \\
\text { tendrán un } \\
\text { funcionamiento menos } \\
\text { óptimo, ocasionando } \\
\text { posiblemente } \\
\text { distracciones, conflictos a } \\
\text { la hora de buscar un } \\
\text { consenso y participación } \\
\text { desigual por parte del } \\
\text { alumnado" }\end{array}$ & $\begin{array}{l}\text { "Los alumnos de } \\
\text { secundaria se cansarían de } \\
\text { tener que tomar tantas } \\
\text { notas y dedicar tanto } \\
\text { tiempo a la observación } \\
\text { sin conseguir llegar a la } \\
\text { identificación de las } \\
\text { plantas. En general, los } \\
\text { estudiantes tienen } \\
\text { obsesión por etiquetar y } \\
\text { poner nombre a todo. Si } \\
\text { no es aś, parece que el } \\
\text { trabajo realizado no tiene } \\
\text { sentido". }\end{array}$ & $\begin{array}{l}\text { "Se habrían dispersado al } \\
\text { no tener un objetivo } \\
\text { claro. Pérdida de interés } \\
\text { al no realizar una } \\
\text { identificación correcta } \\
\text { por no tener suficiente } \\
\text { información" }\end{array}$ \\
\hline
\end{tabular}

Otro aspecto a considerar en relación a las dificultades actitudinales declaradas es que, a pesar de ser un aspecto ampliamente extendido en las respuestas cuando se hace referencia a los alumnos de secundaria, cuando los estudiantes hablan de las dificultades vividas, en ningún momento señalan problemas de este tipo. Es decir, no piensan que ellos mismos tienen 
problemas actitudinales. No obstante, dichas dificultades se observan en las grabaciones de vídeo y son descritas por los estudiantes cuando analizan las actuaciones de sus compañeros (C2CI y C2CC).

\section{Las propuestas de mejora planteadas y el tipo de planteamientos}

Con una representación similar a la del apartado anterior, se analizan los cambios en las propuestas de mejora (figura 4, fila superior) y el tipo de planteamientos presentados (figura 4, fila inferior). Para estos últimos se ha considerado la descripción de la mejora, el número de razones para la mejora planteada y la existencia o no de justificaciones para dichas razones, utilizando círculos coloreados cuyo diámetro es proporcional a la totalidad de propuestas de mejoras dadas en cada uno de los momentos. Asimismo, se ha tenido en consideración la proporción de razones dadas para una determinada propuesta de mejora y si estas, a su vez, son apoyadas con una justificación.

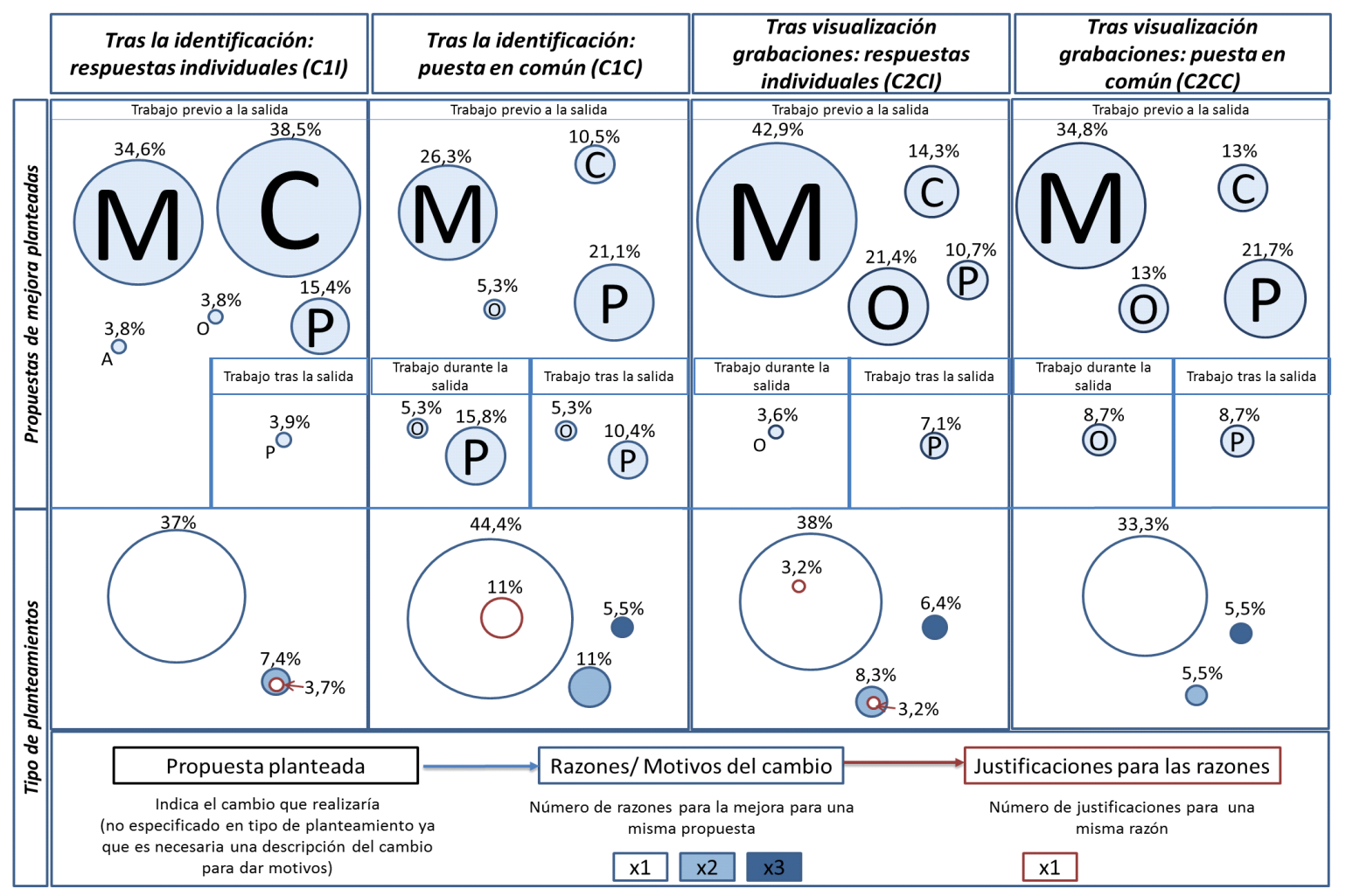

Figura 4. Propuestas de mejora planteadas por los estudiantes de máster en los cuatro momentos y tipo de planteamientos (razones y justificaciones de dichos cambios). Códigos, véase tabla 3.

Hay que señalar que, para las propuestas de mejora y sus planteamientos, las cuestiones a las que debían dar respuesta los estudiantes tenían connotaciones diferentes y ello puede ser el motivo de los resultados obtenidos, sobre todo en lo relacionado con el tipo de planteamientos. En concreto, las cuestiones planteadas en C1I y C2CI demandaban el modo en el que realizarían la actividad en un aula de secundaria. La cuestión demandada en C1C pedía el tipo de propuestas de mejora y su justificación y en el caso del C2CC se pedía únicamente la enumeración de las principales propuestas de mejora. 


\section{Las propuestas de mejora planteadas}

Los elementos a los que hacen referencia los estudiantes cuando plantean propuestas de mejora de la actividad en el contexto de secundaria se modifican en proporción y enfoque a lo largo de la misma. Inicialmente, priorizan la necesidad de explicaciones teóricas previas (C) y la modificación de materiales (M). Tras la puesta en común, los estudiantes consideran cambios en los tres momentos de la salida: antes, durante y después. Asimismo, se reducen las alusiones a explicaciones teóricas previas al mismo tiempo que se incrementan aspectos relativos a la organización $(\mathrm{O})$, como por ejemplo, explicar a los alumnos en qué consiste la actividad o considerar la presencia de más profesores durante la salida.

Tras la visualización de las grabaciones, apenas se detectan cambios entre el tipo de respuestas individuales y las obtenidas tras la puesta en común. No obstante, con respecto a las primeras, se incrementa la importancia de los aspectos organizativos (O) de la actividad en los momentos antes y durante. En estos momentos dan importancia a la explicitación de los objetivos a los estudiantes de secundaria, ir a "visitar la zona donde se va a realizar la actividad antes de realizarla" (como profesores) o "presentar la actividad" a los estudiantes (explicar qué van a hacer).

Tras la salida, las propuestas se centran en trabajar los aspectos procedimentales (P), tales como el uso de la clave de identificación, o trabajar aspectos metacognitivos a través de la utilización de estrategias para la autoevaluación del proceso.

En cuanto a la modificación de los materiales (M), en todos los momentos están de acuerdo en la necesidad de un cambio, bien del glosario de términos, del libro de identificación o de la selección de ejemplares para que el libro usado por sus futuros alumnos contenga dichos ejemplares.

\section{Tipo de planteamientos de las propuestas de mejora}

El tipo de planteamientos dados por los estudiantes sufre pequeñas variaciones a lo largo de los distintos momentos. Se observa un mayor cambio en C1C y, a partir de ese momento, el tipo de planteamientos permanece prácticamente estable, reduciéndose en C2CC. Este hecho sucede cuando se les pide únicamente enumerar las principales propuestas de mejora y, a pesar de ello, el número de motivos dados para los cambios se incrementa.

Inicialmente, los estudiantes (C1I) aportan alguna razón para el cambio propuesto o llegan a dar más de una motivo, siendo capaces de justificar de modo muy superficial solo en una ocasión. Tras la puesta en común, se dan hasta tres motivos por los cuales realizarían ese cambio y justifican en más ocasiones, aunque de un modo también muy superficial. Este aspecto se mantiene en las respuestas individuales tras la visualización de las grabaciones. En la tabla 5 mostramos un ejemplo de propuestas de mejora razonadas y justificadas para los tres primeros momentos y los motivos que dan en el cuarto momento.

Las propuestas planteadas por los tres equipos de trabajo en el último momento suponen una reunificación de propuestas anteriores con algún pequeño cambio de las respuestas dadas en C1C y C2CI. De hecho, en el ejemplo mostrado en la tabla 6 , algunas mejoras se plantean en primera persona a pesar de ser una respuesta dada en grupo (C2CC). 
Tabla 6. Ejemplos de propuestas de mejora planteadas. Las tonalidades azules corresponden a motivos dados por los estudiantes y las marrones a justificaciones de dichos motivos (atendiendo al número de motivos que aparecen en esta respuesta siguiendo la estructura de la figura 3).

\begin{tabular}{|c|c|c|c|}
\hline $\begin{array}{l}\text { Respuesta inicial } \\
\text { (cuestionario C1I) }\end{array}$ & $\begin{array}{c}\text { Puesta en común } \\
\text { inicial (C1C) }\end{array}$ & $\begin{array}{c}\text { Respuesta tras la } \\
\text { visualización de las } \\
\text { grabaciones } \\
\text { (cuestionario C2CI) }\end{array}$ & $\begin{array}{c}\text { Puesta en común } \\
\text { final (C2CC) }\end{array}$ \\
\hline $\begin{array}{l}\text { "Es necesaria una clase } \\
\text { de botánica previa } \\
\text { para que la práctica se } \\
\text { centre en manejar las } \\
\text { claves con cierta } \\
\text { soltura que, a su vez } \\
\text { servirá de repaso de la } \\
\text { teoría y (porque) así se } \\
\text { afianzarán mucho } \\
\text { mejor los conceptos" }\end{array}$ & $\begin{array}{l}\text { "Elaborar unas bases } \\
\text { de orientación } \\
\text { ilustradas con objeto } \\
\text { de facilitar la tarea } \\
\text { descriptiva de los } \\
\text { elementos } \\
\text { considerados en la } \\
\text { clave dicotómica, } \\
\text { dándoles una muestra } \\
\text { muy visual de en qué } \\
\text { consisten las etiquetas } \\
\text { empleadas a lo largo } \\
\text { de la misma. (Ya que) } \\
\text { esto les permitirá } \\
\text { identificar y describir } \\
\text { más precisamente los } \\
\text { elementos } \\
\text { considerados a lo largo } \\
\text { de la clave, evitando } \\
\text { confusiones y pérdidas } \\
\text { de orientación" }\end{array}$ & $\begin{array}{l}\text { "Selección previa de } \\
\text { ejemplares por parte } \\
\text { del profesor para que } \\
\text { no conlleven } \\
\text { dificultades. } \\
\text { Considerando } \\
\text { (porque) se va a } \\
\text { realizar en un grupo de } \\
\text { primero de la ESO." }\end{array}$ & $\begin{array}{l}\text { "Por último, } \\
\text { realizaríamos la } \\
\text { actividad (durante la } \\
\text { actividad). Estaría } \\
\text { cerca de ellos para } \\
\text { asegurarme de que } \\
\text { trabajan, por si tienen } \\
\text { dudas o dificultades y } \\
\text { guiando cada paso si } \\
\text { es necesario. }\end{array}$ \\
\hline
\end{tabular}

\section{Discusión y consideraciones finales}

A partir de los resultados obtenidos, la valoración global del cambio en las ideas de los estudiantes sobre los aspectos didácticos de la salida es positiva, ya que llegan a hacer explícitos muchos de los elementos del CDC teóricamente implicados en este tipo de actividades. Sin embargo, la gráfica imaginaria que podríamos trazar sobre estos cambios llevaría una trayectoria con una gran pendiente inicial que se estabiliza o incluso decrece antes de lo deseable.

En los momentos 1 y 2 (figura 2), donde los estudiantes ponen en común sus ideas iniciales (1) y realizan el análisis de las grabaciones de vídeo de forma individual (2) se observan cambios más o menos relevantes en las ideas expresadas en sus respuestas. En el momento 3, cuando ponen en común los análisis de los vídeos, el tipo de respuestas dadas es muy similar.

En el momento 2, los estudiantes pasan de percibir el objetivo de la propuesta como un modo de aprender conceptos y desarrollar destrezas para la identificación de ejemplares (C1C) a declarar que el objetivo está relacionado con ponerse en el papel del alumno o pensar en las diferentes posibilidades de aplicación de la salida (C2AI). Así, algunos de los estudiantes buscan recetas perfectas de enseñanza, mientras que otros asumen que no existe una receta concreta y que, dependiendo del contexto, una misma actividad puede funcionar mejor de un modo o de otro (véase tabla 4). De este modo, parece ser que algunos de los estudiantes en ese momento de la actividad ya no buscan respuestas “categóricas” (Perrenoud 2004).

Esta percepción del objetivo de la actividad comienza a hacerse visible cuando los estudiantes, 
acuerdan sus respuestas en C1C. Y este cambio, en el momento 1, sucede con el resto de elementos considerados en el análisis (dificultades en secundaria y propuestas de mejora, además de los objetivos). Podríamos decir que cuando los estudiantes trabajan en el aula, poniendo en común y debatiendo sus puntos de vista iniciales, se produce un cambio en sus ideas sobre la dimensión didáctica de la actividad en un sentido similar al indicado por Blomberg et al. (2011).

Las cuestiones relativas a las dificultades vividas y las estrategias de resolución empleadas para resolverlas buscaban la proximidad, la cercanía, lo basado en la propia experiencia (Bhattachryya et al. 2009). Aunque las ideas sobre estos elementos apenas cambian, éstas sirven de apoyo para dar respuesta a las cuestiones que se alejan de la propia experiencia. Es decir, teóricamente sirven de orientación para transferir su experiencia como alumnos a una posible situación de aula como profesores en el momento que plantean las propuestas de mejora.

Sin embargo, esta situación no se observa inicialmente cuando los estudiantes piensan en las posibles dificultades que podría tener un alumno de secundaria. En el cuestionario inicial respondido individualmente $(\mathrm{C} 1 \mathrm{I})$, los problemas asociados a una supuesta "mala selección de materiales" no supondrían una dificultad para los estudiantes de secundaria, mientras que ellos declaran haberlos vivido. No obstante, cuando visualizan las grabaciones de vídeo (a partir del momento 2), se observa un cambio en este sentido: en sus problemas le dan menos importancia a la mala selección de materiales o recursos (o a las limitaciones de los mismos), mientras que cuando se trata de los alumnos de secundaria, sí se tienen en consideración.

Las dificultades actitudinales se presuponen en todos los momentos para los alumnos de secundaria, pero no son declaradas cuando los estudiantes de máster participantes en este estudio hablan de los problemas vividos durante la tarea de identificación a pesar de haber quedado registrados en los vídeos. No obstante, en el caso de las posibles dificultades que podrían presentarse en un aula de secundaria, el sentido de las declaraciones en los últimos momentos se asocia, generalmente, a la aparición de actitudes negativas debido a la dificultad de la tarea o de los materiales utilizados (posible frustración por no alcanzar una meta).

En el conjunto de las declaraciones relativas a las posibles dificultades de los alumnos de secundaria se observa cierto equilibrio: conforme aparecen dificultades asociadas a los materiales, no declaradas en los primeros momentos, las dificultades actitudinales declaradas cambian también en el mismo sentido (si el material tiene problemas, los estudiantes tienen problemas).

La falta de declaración de dificultades actitudinales por parte de los estudiantes de máster durante su actuación podría asociarse a un modo de protección tanto de su equipo de trabajo como de su papel como estudiantes. Al fin y al cabo, son estudiantes y actúan como tales. O bien, puede deberse a no haber dado importancia a dichos aspectos, ya que finalmente han resuelto la mayor parte de sus problemas y, por ello, no haberlos considerado. No obstante, habría sido necesaria la realización de entrevistas o grupos de discusión para conocer los motivos reales de lo que podríamos considerar como ausencia de autocrítica sobre esos aspectos.

Los problemas previstos en secundaria se utilizan cuando plantean las propuestas de mejora. Se trata de una vía que los estudiantes de máster utilizan para dar razones por las que realizarían la actividad de ese modo. En este aspecto los cambios se hacen relevantes en el momento 1, donde consideran tres fases para la actividad (antes, durante y después de la salida) y comienzan a tener presente la importancia del papel del profesor en el proceso (sobre lo que tiene que saber o cómo debe actuar en momentos en los que los alumnos están en un espacio abierto y poco controlable). Estas consideraciones se hacen más patentes todavía en el 
momento 2, llegando a considerar: 1) una preparación previa a la salida tanto por parte del profesor (conocer el lugar antes de hacer la actividad) como por parte del alumno (explicar en qué consiste la salida, cuales son los objetivos), 2) qué hacer durante la salida para solucionar problemas como la falta de focalización en la observación y 3) cómo hacer que los estudiantes no acaben la actividad en el campo a través del uso de estrategias que ayuden a hacerles pensar sobre lo que han aprendido. Estaríamos hablando de las recomendaciones expuestas por diferentes autores sobre los objetivos del trabajo de campo (Orion y Hofstein 1994 o Pedrinaci et al 1994, por ejemplo). A pesar de que estas nociones fueron expuestas en la primera sesión de la actividad, no es hasta la puesta en común cuando comienzan a emerger y a tener sentido para ellos como futuros docentes. Es decir, parece necesaria esa reflexión colectiva para que se hagan explícitos determinados aspectos ligados al proceso de enseñanza y aprendizaje de las ciencias en contextos prácticos (Yoon y Kim 2010).

En cuanto al tipo de planteamientos en las propuestas de mejora, no se ha logrado consolidar una estrategia de análisis que nos permita visibilizar grandes cambios. Se reconoce un incremento en el tipo de motivos por los que se realiza una propuesta concreta, pero las justificaciones son vagas y aparecen de forma similar en cada uno de los documentos analizados. Tal vez el modo en el que se plantearon las cuestiones en relación a las propuestas de mejora facilita la recogida de datos pero a cambio no permite estudiar en profundidad el modo en el que se argumentan los cambios propuestos.

En este caso, no solo en las propuestas de mejora, sino también en las posibles dificultades que piensan que podrían tener los alumnos de secundaria, se observa un cambio en los motivos que presentan los estudiantes del máster, así como en su profundidad, posicionándose en un sentido más próximo al papel del profesor y más alejado de su papel de estudiantes.

En cualquier caso, existen indicios que parecen evidenciar una mayor reflexión en sus ideas en los últimos momentos, ya que, por un lado, retoman sus conocimientos sobre la experiencia vivida y los usan para predecir dificultades en los alumnos de secundaria y plantear cambios en la propuesta y, por otro, algunos de los estudiantes pasan de ver la actividad desde el papel de "estudiantes de máster" al de "profesores en formación inicial". Así, estos hechos permiten destacar la importancia de: 1) continuar con actividades que promuevan la discusión grupal y el análisis de las actividades realizadas tras la realización de secuencias transferibles a un aula de secundaria; 2) promover el uso de pequeños grupos de trabajo para discutir sobre la actividad realizada tras una reflexión individual previa y 3) facilitar el análisis de las actividades a través de las grabaciones de vídeo para favorecer un cambio en las ideas "didácticas" iniciales.

\section{Agradecimientos}

Grupo de referencia BEAGLE Investigación en Didáctica de las Ciencias Naturales (Gobierno de Aragón y Fondo Social Europeo) Instituto de Investigación en Ciencias Ambientales de Aragón (IUCA/UNIZAR). Proyecto EDU2016-76743-P(MINECO). Agradecemos al editor y a los revisores anónimos sus aportaciones para la mejora del trabajo.

\section{Referencias}

Acevedo J.A. (2009). Conocimiento didáctico del contenido para la enseñanza de la naturaleza de la ciencia (I). Revista Eureka sobre Enseñanza y Divulgación de las Ciencias, 6(1), 21-46. http://doi.org/10.25267/Rev_Eureka_ensen_divulg_cienc.2009.v6.i1.02

Aguilera D. (2018) La salida de campo como recurso didáctico para enseñar ciencias. Una revisión sistemática. Revista Eureka sobre Enseñanza y Divulgación de las Ciencias 15(3), 3103-1-17. http://doi.org//10.25267/Rev_Eureka_ensen_divulg_cienc.2018.v15.i3.3103 
Allen C.D. (2011). Concept mapping validates fieldwork's capacity to deepen students' cognitive linkages of complex processes. Research in Geographic Education, 13, 30-51.

Amórtegui E. Gavidia V. y Mayoral O. (2016). Las prácticas de campo en la enseñanza de la Biología y la formación docente: Estado actual del conocimiento. En J.L. Bravo Galán (ed.), 27 Encuentros de Didáctica de las Ciencias Experimentales (pp. 175-182). Badajoz: Universidad de Extremadura.

Bebbington A. (2005). The ability of A-level students to name plants. Journal of Biological Education, 39(2), 63-67. http://doi.org/10.1080/00219266.2005.9655963

Behrendt M. y Franklin T. (2014). A Review of Research on School Field Trips and Their Value in Education. International Journal of Environmental and Science Education, 9(3), 235245. http://doi.org/10.12973/ijese.2014.213a

Benedict-Chambers A. (2016). Using tools to promote novice teacher noticing of science teaching practices in post-rehearsal discussions. Teaching and Teacher Education, 59, 28-44. http://doi.org/10.1016/j.tate.2016.05.009.

Bhattacharyya S., Volk T. y Lumpe A. (2009). The Influence of an Extensive Inquiry-Based Field Experience on Pre-Service Elementary Student Teachers' Science Teaching Beliefs. Journal of Science Teacher Education, 20, 199-218. https://doi.org/10.1007/s10972-009-9129-8

Blomberg G., Gamoran Sherin M., Renkl A., Glogger I. y Seidel T. (2014). Understanding video as a tool for teacher education: investigating instructional strategies to promote reflection. Instructional Science, 42, 443-463. http://doi.org/10.1007/s11251-013-9281-6.

Blomberg G., Stürmer K. y Seidel T. (2011). How pre-service teachers observe teaching on video: Effects of viewers' teaching subjects and the subject of the video. Teaching and Teacher Education, 27, 1131-1140. https://doi.org/10.1016/j.tate.2011.04.008

Braund M. y Reiss M. (2006). Towards a more authentic science curriculum: the contribution of out-of-school learning. International Journal of Science Education, 28, 1373-1388. https://doi.org/10.1080/09500690500498419

Brusi D., Zamorano M., Casellas R.M. y Bach J. (2011). Reflexiones sobre el diseño por competencias en el trabajo de campo en Geología. Enseñanza de las Ciencias de la Tierra, 19(1), 4-14.

Carrasquer J., Álvarez M.V., Lafuente A. y Pérez I. (2001). Nuestros amigos los árboles y arbustos $\left(2^{a}\right.$ edición). Teruel: Excmo. Ayuntamiento de Teruel.

Del Carmen L. y Pedrinaci E. (1997). El uso del entorno y del trabajo de campo. En L. del Carmen (Coord.). La enseñanza y aprendizaje de las ciencias de la naturaleza en educación secundaria (pp. 133-154). Cuadernos de Formación del Profesorado, 9. Barcelona: ICE, Universitat de Barcelona-Horsori.

DeWitt J. y Storksdieck M. (2008). A short review of school field trips: Key findings from the past and implications for the future. Visitor Studies, 11(2), 181-197. https://doi.org/10.1080/10645570802355562

Dickerson D., Dawkins K. y Annetta L. (2007). Scientific Fieldwork: An Opportunity for Pedagogical-Content Knowledge Development. Journal of Geoscience Education, 55(5), 371-376. https://doi.org/10.5408/1089-9995-55.5.371 
Dillon J., Rickinson M., Teamey K., Morris M., Choi M.Y., Sanders D. y Benefield, P. (2006). The value of outdoor learning: evidence from research in the UK and elsewhere. School Science Review, 87, 107-111.

Dori Y.J. y Herscovitz O. (2005). Case-based long-term professional development of science teachers. International Journal of Science Education, 27(12), 1413-1446. https://doi.org/10.1080/09500690500102946

Gess-Newsome J. (1999). Pedagogical content knowledge: an introduction and orientation. En J. Gess-Newsome y N.G. Lederman (Eds.), Examining pedagogical content knowledge: the construct and its implications for science education (pp. 3-17). Dordrecht, The Netherlands: Kluwer Academic Publishers.

Hatch T., Shuttleworth J., Jaffee A.T. y Marri A. (2016). Videos, pairs, and peers: What connects theory and practice in teacher education? Teaching and Teacher Education, 59, 274-284. https://doi.org/10.1016/j.tate.2016.04.011

Hatton N. y Smith D. (1995). Reflection in teacher education: Towards definitions and implementation. Teaching and Teacher Education, 11, 33-49. https://doi.org/10.1016/0742-051X(94)00012-U

Mena M., García Rodriguez M.L. y Tillema H. (2012). Student teacher reflective writing: What does it reveal? European Journal of Teacher Education, 1, 1-17. http://doi.org/10.1080/02619768.2012.713933

Morag O. y Tal T. (2012) Assessing Learning in the Outdoors with the Field Trip in Natural Environments (FiNE) Framework. International Journal of Science Education, 34(5), 745777. http:/ / doi.org/10.1080/09500693.2011.599046

Nilsson P. (2008). Teaching for understanding: The complex nature of pedagogical content knowledge in pre-service education. International Journal of Science Education, 30(10), 1281-1299. https://doi.org/10.1080/09500690802186993

Olson J.K., Cox-Petersen A.M. y McComas W.F. (2001). The inclusion of informal environments in science teacher preparation. Journal of Science Teacher Education, 12, 155-173. https://doi.org/10.1023/A:1016715127697

Orion N. y Hofstein A. (1994). Factors that influence learning during a scientific field trip in a natural environment. Journal of Research in Science Teaching, 31, 1097-1119. https://doi.org/10.1002/tea.3660311005

Park S. y Oliver J.S. (2008). Revisiting the Conceptualisation of Pedagogical Content Knowledge (PCK): PCK as a Conceptual Tool to Understand Teachers as Professionals. Research on science education, 38, 261-284. https://doi.org/10.1007/s11165007-9049-6

Pedrinaci E. (2012). Trabajo de campo y aprendizaje de las ciencias. Alambique. Didáctica de las Ciencias Experimentales, 71, 81-89.

Pedrinaci E., Sequeiros L. y García de la Torre E. (1994). El trabajo de campo y el aprendizaje de la Geología. Alambique. Didáctica de las Ciencias Experimentales, 2, 37-46.

Perrenoud P. (2004). Desarrollar la práctica reflexiva en el oficio de enseñar. Barcelona: Graó.

Rich P.J. y Hannafin M. (2009). Technologies to Scaffold, Structure, and transform Teacher Reflection. Journal of Teacher Education, 60(1), 52-67. https://doi.org/10.1177/0022487108328486 
Rickinson M., Dillon J., Teamey K., Morris M., Choi M.Y., Sanders D. y Benefield P. (2004). A Review of Research,on Outdoor Learning. Shrewsbury, UK: National Foundation for Educational Research and King's College.

Sáez Bondía, M.J. y Cortés Gracia, A.L. (2012). Dificultades en la construcción del conocimiento científico detectadas analizando grabaciones de una actividad con maestros en formación. En J.M. Domínguez Castiñeiras (ed.), XXV Encuentro de Didáctica de las Ciencias Experimentales (pp. 601-608), Santiago de Compostela: USC/APICE.

Sáez Bondía, M.J. y Cortés Gracia, A.L. (2014). El trabajo de campo en contextos de indagación. Estudio comparativo en la formación inicial del profesorado de primaria y secundaria. En M.A. de las Héras Pérez, A.A. Lorca Martí, B. Vázquez Bernal, A.M. Wamba Aguado y R. Jiménez Pérez (eds.), Investigación y transferencia para una educación en ciencias: Un reto emocionante (pp. 184-192). Huelva: Servicio de Publicaciones. Universidad de Huelva.

Sáez Bondía, M.J. y Cortés Gracia, A.L. (2016). Poniendo en apuros al profesorado en formación como paso previo a la reflexión. En J.L. Bravo Galán (coord.), 27 Encuentros de Didáctica de las Ciencias Experimentales (pp. 379-386). Badajoz: Universidad de Extremadura y APICE.

Shulman L.S. (1986). Those who understand: knowledge growth in teaching. Educational Researcher, 15(2), 4-14. https://doi.org/10.3102/0013189X015002004

Simon S. y Campbell S. (2012). Teacher learning and professional development in science education. En B. Fraser, K. Tobin y McRobbie C.J. (Eds.) Second international bandbook of science education (pp. 307-321). Springer Netherlands.

Simons H. (2011). El estudio de caso: teoría y práctica. Madrid: Morata.

Smith D. (1991). Educating the reflective practitioner in curriculum. Curriculum, 12, 115-124.

Yin R.K. (2014). Case Study Research. Design and methods. Thousand Oaks: SAGE Publications.

Yoon H.G. y Kim M. (2010). Collaborative reflection through dilemma cases of science practical work during practicum. International Journal of Science Education, 32(3), 283-301. https://doi.org/10.1080/09500690802516538 